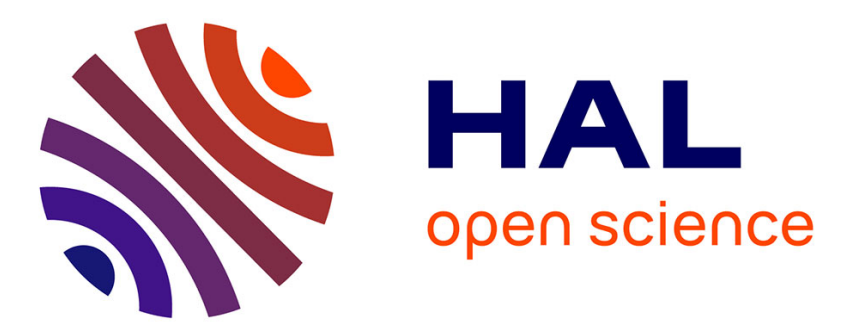

\title{
Midinfrared frequency combs from coherent supercontinuum in chalcogenide and optical parametric oscillation
}

Kevin F. Lee, Nicolai Granzow, Markus A. Schmidt, W. Chang, L. Wang, Quentin Coulombier, Johann Troles, N. Leindecker, K. Vodopyanov, P. G. Schunemann, et al.

\section{To cite this version:}

Kevin F. Lee, Nicolai Granzow, Markus A. Schmidt, W. Chang, L. Wang, et al.. Midinfrared frequency combs from coherent supercontinuum in chalcogenide and optical parametric oscillation. Optics Letters, 2014, 39 (7), pp.2056-2059. 10.1364/OL.39.002056 . hal-01061285

\section{HAL Id: hal-01061285 \\ https://hal.science/hal-01061285}

Submitted on 8 Sep 2014

HAL is a multi-disciplinary open access archive for the deposit and dissemination of scientific research documents, whether they are published or not. The documents may come from teaching and research institutions in France or abroad, or from public or private research centers.
L'archive ouverte pluridisciplinaire HAL, est destinée au dépôt et à la diffusion de documents scientifiques de niveau recherche, publiés ou non, émanant des établissements d'enseignement et de recherche français ou étrangers, des laboratoires publics ou privés. 


\title{
Midinfrared frequency combs from coherent supercontinuum in chalcogenide and optical parametric oscillation
}

\author{
Kevin F. Lee, ${ }^{1, *}$ N. Granzow, ${ }^{2}$ M. A. Schmidt, ${ }^{2,3}$ W. Chang,,${ }^{2,4}$ L. Wang, ${ }^{2}$ Q. Coulombier, ${ }^{5}$ J. Troles, ${ }^{6}$ \\ Nick Leindecker ${ }^{1,7}$ Konstantin L. Vodopyanov, ${ }^{8}$ Peter G. Schunemann, ${ }^{9}$ \\ M. E. Fermann, ${ }^{1}$ P. St. J. Russell, ${ }^{2}$ and I. Hartl ${ }^{10}$ \\ ${ }^{1}$ IMRA America, Inc., 1044 Woodridge Ave., Ann Arbor, Michigan 48105, USA \\ ${ }^{2}$ Max Planck Institute for the Science of Light, Guenther-Scharowsky-Str. 1, 91058 Erlangen, Germany \\ ${ }^{3}$ Institute of Photonic Technology, Albert-Einstein-Str. 9, 07745 Jena, Germany \\ ${ }^{4}$ Optical Sciences Group, Research School of Physics and Engineering, The Australian National University, \\ Canberra 0200 ACT, Australia \\ ${ }^{5}$ Laboratoire PhLAM, CNRS/Université de Lille1, UMR8523, 59658 Villeneuve d'Ascq Cedex, France \\ ${ }^{6}$ Sciences Chimiques de Rennes, Université de Rennes I, 35042 Rennes, France \\ ${ }^{7}$ E. L. Ginzton Laboratory, Stanford University, Stanford, California 94305, USA \\ ${ }^{8}$ CREOL, College of Optics and Photonics, University of Central Florida, Orlando, Florida 32816, USA \\ ${ }^{9}$ BAE Systems, P.O. Box 868, Nashua, New Hampshire 03063, USA \\ ${ }^{10}$ Deutsches Elektronen-Synchrotron (DESY), Notkestrasse 85, 22607 Hamburg, Germany \\ *Corresponding author: klee@imra.com
}

Received January 13, 2014; revised February 28, 2014; accepted March 1, 2014; posted March 3, 2014 (Doc. ID 204619); published March 26, 2014

\begin{abstract}
We observe the coherence of the supercontinuum generated in a nanospike chalcogenide-silica hybrid waveguide pumped at $2 \mu \mathrm{m}$. The supercontinuum is shown to be coherent with the pump by interfering it with a doubly resonant optical parametric oscillator (OPO) that is itself coherent with the shared pump laser. This enables coherent locking of the OPO to the optically referenced pump frequency comb, resulting in a composite frequency comb with wavelengths from 1 to $6 \mu \mathrm{m}$. (C) 2014 Optical Society of America

OCIS codes: (060.2390) Fiber optics, infrared; (190.4970) Parametric oscillators and amplifiers; (320.6629) Supercontinuum generation.

http://dx.doi.org/10.1364/OL.39.002056
\end{abstract}

Frequency combs have been reaching beyond visible wavelengths toward the ultraviolet [1] and midinfrared (mid-IR) [2]. Mid-IR combs are being developed to bring precision comb spectroscopy to the molecular fingerprint region and are produced by difference frequency generation, optical parametric oscillators (OPOs), microresonators, and supercontinuum generation. In the latter case, as long as the generated supercontinuum maintains coherence with the pump laser, a device that enables an existing frequency comb to be broadened into the mid-IR (simply by passing through a waveguide) would be ideal for producing broadband mid-IR frequency combs. Since conventional silica fibers absorb wavelengths longer than $2 \mu \mathrm{m}$, they must be replaced by materials such as chalcogenide glasses, which are both transparent in the mid-IR and strongly nonlinear. Chalcogenide fibers

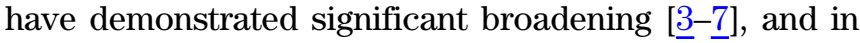
one case have been shown to produce a frequency comb that is coherent with the pump laser [7] , as is required to preserve the frequency comb structure.

Here, we use a hybrid chalcogenide-silica nanospike waveguide [5] to simplify mid-IR frequency comb generation. The waveguide is pumped directly by a $2 \mu \mathrm{m}$ amplified frequency comb, without requiring an intermediate $\mathrm{OPO}$ as in [7], and the waveguide is small and simple, using freespace coupling into and out of the fiber, avoiding the burdens of an in situ tapering apparatus []].

We verify that the supercontinuum generated by the nanospike is coherent by interfering it with the output from a doubly resonant OPO pumped by the same frequency comb, which is known to have a stable frequency relative to its pump []. We observe a strong beat frequency, showing that the supercontinuum is both coherent and maintains the frequency comb structure of the pump. Jittering of this beat note is mostly due to vibrations of the OPO cavity. By stabilizing the OPO cavity length against this beat, the OPO output also becomes a stable frequency comb. This composite comb can access most of the 1-6 $\mu \mathrm{m}$ wavelength range.

The nonlinear waveguide consists of a tapered silica capillary filled with $\mathrm{As}_{2} \mathrm{~S}_{3}$. To create this structure, chalcogenide glass is placed in a larger inner diameter silica capillary, which is fused to the smaller inner diameter target capillary. The fibers are then heated to melt the chalcogenide, which is pushed into the smaller capillary by pressurized argon. By sealing the end of the target capillary before filling, the capillary tapers down to a point, as illustrated in Fig. 1. This inverse-tapered nanospike shape improves the launch efficiency by about 60 times [5].

The final device is $2 \mathrm{~mm}$ long, the first $0.3 \mathrm{~mm}$ of which is the nanospike. The untapered region has an inner diameter of $1 \mu \mathrm{m}$. Unlike other devices based on tapering chalcogenide fiber, where there is significant propagation in unstretched fiber before and after the supercontinuum generating region, the pump light is launched almost immediately into the supercontinuum generating section. This has the significant advantages of minimizing 


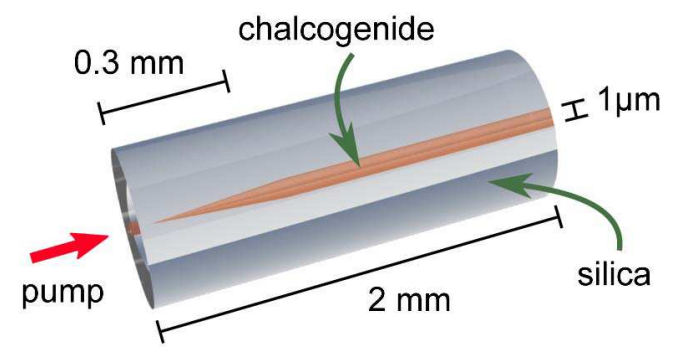

Fig. 1. Illustration of chalcogenide nanospike embedded in silica. Light enters from the tapered side on the left.

unwanted dispersion of the pump pulses and avoiding absorption of the generated light. Being embedded in silica, the chalcogenide glass is protected physically and chemically; unprotected, it is fragile and deteriorates in the presence of moisture. The device can be used for hours to days depending on pumping level, generating spectra down to wavelengths as low as $1.3 \mu \mathrm{m}$ and as high as $3.8 \mu \mathrm{m}$ at energies of up to $18 \mathrm{pJ}$ []ㅡ.

The pump laser is an amplified thulium fiber frequency comb producing $2 \mu \mathrm{m}, 65$ fs pulses at a $98.6 \mathrm{MHz}$ repetition rate, with total power of about $400 \mathrm{~mW}$ [9]. To stabilize the frequency comb, part of the oscillator light is amplified to generate a supercontinuum in nonlinear silica fiber from 1 to $2.3 \mu \mathrm{m}$. The resulting 1.1 and $2.2 \mu \mathrm{m}$ wavelengths are used to measure the carrier envelope offset by f-2f interferometry. The $1.56 \mu \mathrm{m}$ component is interfered with a stable, continuous-wave, reference diode laser. These two beat frequencies are coherently locked to fixed values [9], meaning that the stability of the frequency comb lines is the same as the $3 \mathrm{kHz}$ linewidth optical reference.

In this experiment, the nanospike was driven by $7 \mathrm{~mW}$ of pump light, leaving most of the amplified beam available for driving the OPO. The synchronously pumped, doubly resonant OPO uses orientation-patterned GaAs in a ring cavity [10] to convert the $2 \mu \mathrm{m}$ wavelength pulses to the signal wave centered at $3.25 \mu \mathrm{m}$, and the idler wave centered at $5.3 \mu \mathrm{m}$, with about $10 \mathrm{~mW}$ of total output power. A doubly resonant OPO is interferometrically sensitive and requires cavity-length stabilization. The OPO was stabilized by dither locking a cavity mirror on a piezoelectric stack while monitoring the output intensity [10]. The OPO cavity length and nanospike pump energy were chosen to optimize spectral overlap, as shown in Fig. 2 .

The supercontinuum and OPO output are spatiotemporally combined on a partial reflector. The combined

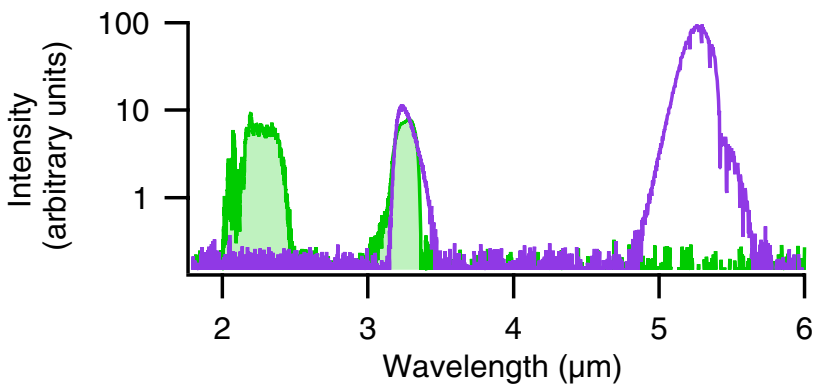

Fig. 2. Optical spectrum of nanospike supercontinuum (filled) and OPO (hollow) when set for overlap at $3 \mu \mathrm{m}$.

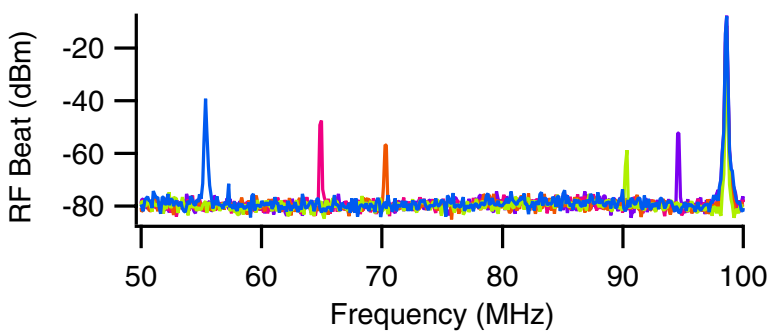

Fig. 3. Measured interference in the radio frequency spectrum of overlapped supercontinuum and dither-locked OPO pulses. The repetition rate is at $98.6 \mathrm{MHz}$; other peaks are beat frequencies. Different colors correspond to different OPO cavity lengths, which have different wavelengths. The presence of strong beats shows that the supercontinuum is coherent.

beam passes through a long-pass filter with $2.5 \mu \mathrm{m}$ cutoff to remove pump light and is measured on a fast liquidnitrogen-cooled InSb detector. Measured radio frequency spectra at a resolution bandwidth of $50 \mathrm{kHz}$ are shown in Fig. 3, the different curves corresponding to different $\mathrm{OPO}$ cavity lengths. For each curve, there is the expected repetition rate peak at $98.6 \mathrm{MHz}$ and also a strong beat frequency with up to $40 \mathrm{~dB}$ signal-to-noise ratio. The beat frequency was verified to exist only near zero time delay between the supercontinuum and OPO. The frequency of the beat was also seen to vary as a function of OPO cavity length. For a doubly resonant OPO, the dither lock can stabilize the cavity at a few discrete cavity lengths that have slightly different spectra and carrier envelope offset frequencies [8]. As the OPO signal wave carrier envelope offset frequency is changed, the beat frequency will also change.

The strong interference between the supercontinuum and the OPO shows that the supercontinuum generation process in the nanospike waveguide is coherent. We have simulated the supercontinuum generation process in a chalcogenide nanospike waveguide and find that we expect most of the light generated to be highly coherent with the pump laser, even when generating the full spectrum out to $4 \mu \mathrm{m}$ as in [5], as shown in Figs. 4(b) and $\underline{4(\mathrm{c})}$.

The simulations include fiber core mode attenuation, dispersion, and effective mode areas within the nanospike (150-500 nm core radius for $0.21 \mathrm{~mm}$ length) and constant diameter (500 $\mathrm{nm}$ radius for the $1.7 \mathrm{~mm}$ length) section. The nonlinear coefficient decreases toward longer wavelengths as shown in Fig. 4(a), a result of the increasing effective mode area. We used a constant value of $2.9 \times 10^{-18} \mathrm{~m}^{2} / \mathrm{W}$ for the nonlinear refractive index of $\mathrm{As}_{2} \mathrm{~S}_{3}$, which is a reasonable assumption since all relevant electronic resonances are in the visible spectral regime. Since the mode in the constant diameter section is mostly located inside the chalcogenide core (the core/ cladding Poynting vector fraction at $2 \mu \mathrm{m}$ wavelength is $90 \%$ ), a possible contribution of the silica nonlinear refractive index can be neglected. For all wavelengths considered here, the modal attenuation is below $1 \mathrm{~dB} / \mathrm{mm}$ (the loss of the fundamental mode is shown in Fig. 2(b) of Ref. [5]). The input was assumed to be a transform-limited Gaussian pulse centered at $1950 \mathrm{~nm}$ with 65 fs full width at half-maximum duration and $18 \mathrm{pJ}$ energy. The coherence properties were quantified by performing multiple simulations with random 
(a)

(b)

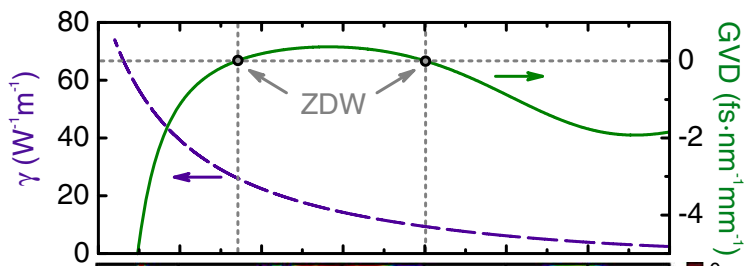

(c)
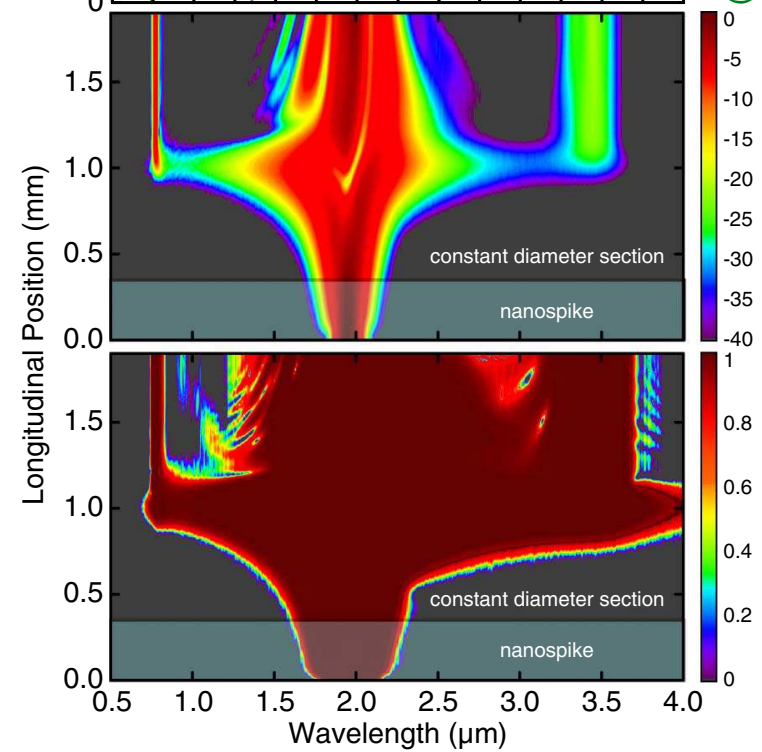

Fig. 4. Simulation of optical properties and supercontinuum generation inside the chalcogenide-silica waveguide. (a) Spectral dependence of nonlinear coefficient (dashed purple curve) and group velocity dispersion (solid green curve) within the constant diameter section. The two zero-dispersion wavelengths (ZDWs) are highlighted by the two grey dotted vertical lines. (b) Generated supercontinuum spectrum at different longitudinal positions within the fiber (color scale is the normalized spectrum in decibels). (c) Corresponding degree of firstorder coherence (color scale is linear, 1 is full coherence with the pump laser). The region of propagation within the nanospike is indicated by the light grey sections in (b) and (c).

quantum noise on the input pulse and calculating the modulus of the complex degree of first-order coherence [11]. A line plot of the simulated spectrum and coherence at the output of the waveguide is shown in Fig. 5 .

Given the coherence of the supercontinuum, we can immediately take this new source and apply it to the

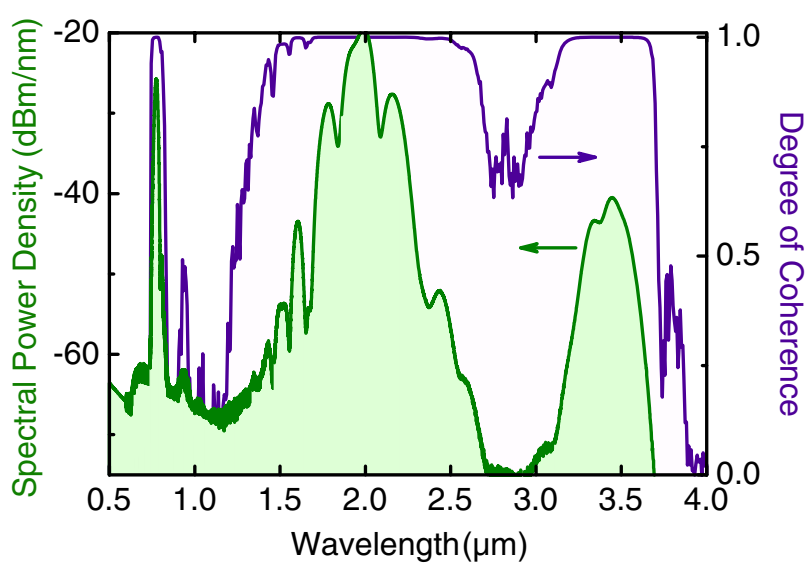

Fig. 5. Simulated spectrum (green curve in front, left axis) and degree of coherence (purple curve behind, right axis) at the output of the chalcogenide-silica waveguide.

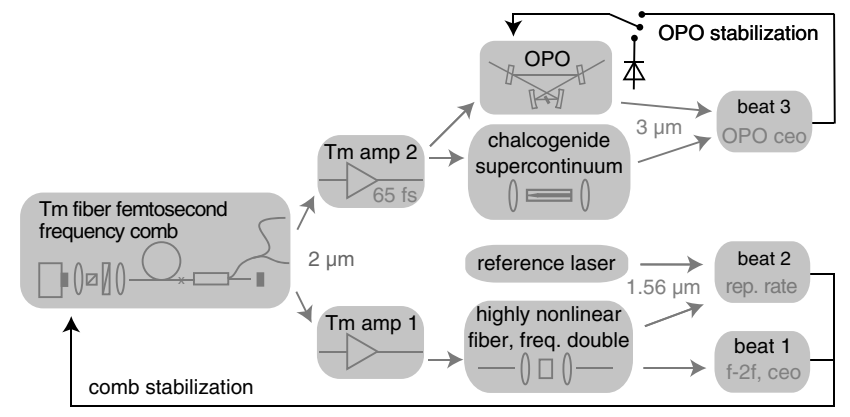

Fig. 6. Schematic of optical and electrical signals for phase locking of the doubly resonant OPO. All the resulting frequency combs in the near and mid-IR are coherently locked to the optical reference.

stabilization of the doubly resonant OPO. While the repetition rate of the OPO follows the pump, changes to the effective cavity length from vibration or crystal heating lead to changes in the output amplitude and the carrier envelope offset of the signal and idler waves. When using a dither lock, the OPO will maintain oscillation but the mid-IR comb is not perfectly phase locked to the pump, with a comb linewidth on the scale of $100 \mathrm{kHz}$ [8]. To improve the stabilization, we use the beat of the OPO with the nanospike supercontinuum.

Since a coherent supercontinuum extends the frequency comb structure of its pump laser, any jitter of the beat of the OPO signal and the supercontinuum indicates a change in the OPO spectrum. Given the constant repetition rate, this is equivalent to measuring changes in the OPO carrier envelope offset. We stabilize this beat frequency by adjusting an OPO cavity mirror mounted on a piezoelectric stack to control the OPO cavity length.

A schematic of this system is shown in Fig. 6. Using this method, we were able to phase lock the OPO to the nanospike supercontinuum. The stability of the locked beat frequency is shown in Fig. 7 , with a relative linewidth lower than our measurement limit of $50 \mathrm{mHz}$. The lock had a cumulative phase noise of $0.68 \mathrm{rad}$, integrated from $100 \mathrm{mHz}$ to $3.5 \mathrm{MHz}$.

The coherent locking of the OPO means that the entire system is coherently locked to the optical reference laser, so all the frequency combs will have the $3 \mathrm{kHz}$ linewidth of the reference laser. The entire system is a

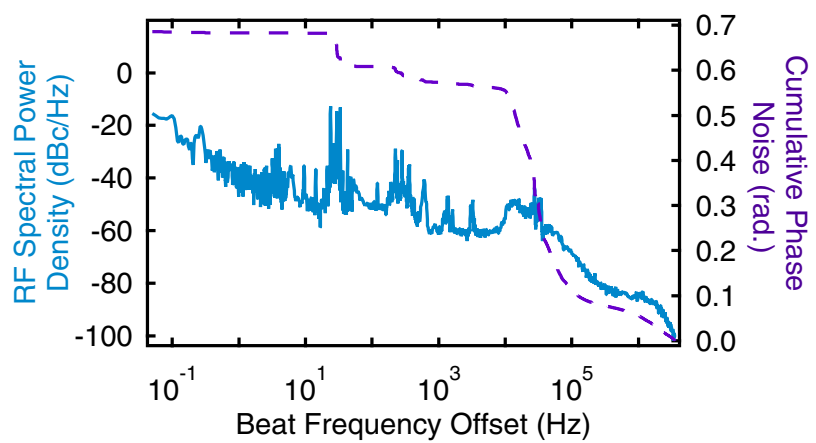

Fig. 7. Plot of the power distribution of the phase-locked beat note between the nanospike supercontinuum and the doubly resonant OPO. The solid curve is the radio-frequency beat, and the dashed curve is the corresponding cumulative phase noise. 
composite of three frequency combs with shared repetition rates and different carrier envelope offset frequencies. The original comb is at $2 \mu \mathrm{m}$, with both silica and nanospike supercontinua extending the range down to $1 \mu \mathrm{m}$ and longer than $3 \mu \mathrm{m}$. The OPO produces a signal frequency comb around $3 \mu \mathrm{m}$ and an idler frequency comb past $5 \mu \mathrm{m}$, with similar OPO systems showing gapless spectra from 2.6 to $6.1 \mu \mathrm{m}$ [10].

In summary, we have shown that the supercontinuum generated by a chalcogenide nanospike waveguide is coherent with the pump oscillator, providing a simple new source of mid-IR frequency combs from 1 to $4 \mu \mathrm{m}$. This nanospike comb can be used to coherently lock the output of a low-threshold doubly resonant OPO, providing a mid-IR comb from 3 to $6 \mu \mathrm{m}$. The OPO wavelength can be readily extended beyond $10 \mu \mathrm{m}$ given the mid-IR transparency of the GaAs crystal used, and the output power increased by using stronger pump lasers. These two new sources are promising candidates for the mid-IR frequency comb sources needed for the coming applications in precision mid-IR spectroscopy.

\section{References}

1. A. Cingöz, D. C. Yost, T. K. Allison, A. Ruehl, M. E. Fermann, I. Hartl, and J. Ye, Nature 482, 68 (2012).
2. A. Schliesser, N. Picque, and T. W. Hänsch, Nat. Photonics 6, 440 (2012).

3. D. D. Hudson, S. A. Dekker, E. C. Mägi, A. C. Judge, S. D. Jackson, E. Li, J. S. Sanghera, L. B. Shaw, I. D. Aggarwal, and B. J. Eggleton, Opt. Lett. 36, 1122 (2011).

4. S. Shabahang, M. P. Marquez, G. Tao, M. U. Piracha, D. Nguyen, P. J. Delfyett, and A. F. Abouraddy, Opt. Lett. 37, 4639 (2012).

5. N. Granzow, M. A. Schmidt, W. Chang, L. Wang, Q. Coulombier, J. Troles, P. Toupin, I. Hartl, K. F. Lee, M. E. Fermann, L. Wondraczek, and P. S. Russell, Opt. Express 21, 10969 (2013).

6. D.-I. Yeom, E. C. Mägi, M. R. E. Lamont, M. A. F. Roelens, L. Fu, and B. J. Eggleton, Opt. Lett. 33, 660 (2008).

7. A. Marandi, C. W. Rudy, V. G. Plotnichenko, E. M. Dianov, K. L. Vodopyanov, and R. L. Byer, Opt. Express 20, 24218 (2012).

8. K. F. Lee, J. Jiang, C. Mohr, J. Bethge, M. E. Fermann, N. Leindecker, K. L. Vodopyanov, P. G. Schunemann, and I. Hartl, Opt. Lett. 38, 1191 (2013).

9. J. Bethge, J. Jiang, C. Mohr, M. Fermann, and I. Hartl, in Lasers, Sources, and Related Photonic Devices (Optical Society of America, 2012), paper AT5A.3.

10. N. Leindecker, A. Marandi, R. L. Byer, K. L. Vodopyanov, J. Jiang, I. Hartl, M. Fermann, and P. G. Schunemann, Opt. Express 20, 7046 (2012).

11. J. M. Dudley and S. Coen, Opt. Lett. 27, 1180 (2002). 